\title{
Worm strains mix but don't match
}

The resources that have made the worm Caenorhabditis elegans a model laboratory organism for developmental genetics research are increasingly proving to be just as useful for studies of natural genetic variation. In a study that started with a skewed Mendelian ratio, H. Seidel, M. Rockman and L. Kruglyak have established, through population genetic and genomic analysis, that many worldwide populations of the worm harbour alleles at two linked loci that are incompatible with each other. The alleles that underlie such detrimental interactions would normally be eliminated from the population but are retained in this instance by balancing selection.

To study natural genetic variation, the authors repeatedly intercrossed

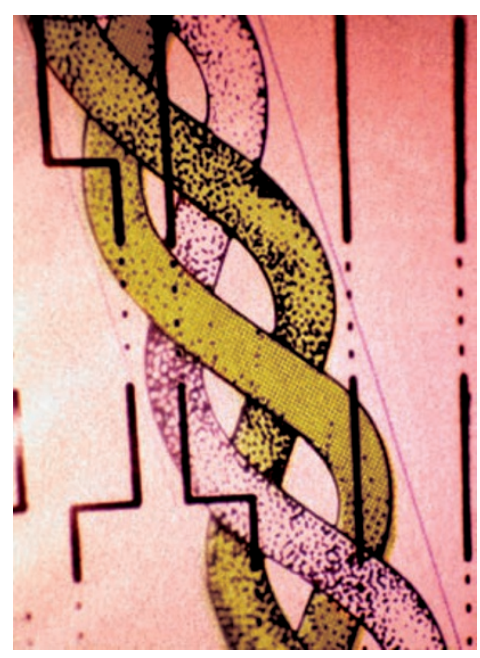

the Bristol strain of C. elegans, the standard laboratory worm, and a Hawaiian strain, CB4856. The descendent strains showed a marked under-representation of Hawaiianstrain alleles in one region of the genome. Crosses revealed that this biased segregation pattern was due to the loss of progeny that are homozygous for the Hawaiian region and that, curiously, the lethality only occurs when the zygote is fertilized by sperm from a worm carrying a Bristol allele at a linked locus. This Bristol allele is not inherited by the dying embryos - it operates through the sperm by paternal effect.

The two loci in question - zeel-1 (zygotic epistatic embryonic lethal 1) and peel-1 (paternal effect epistatic embryonic lethal 1) - were mapped to the same, highly polymorphic 33-kb region. Surprisingly, tight linkage between these alleles persists in all but one of the 62 wild isolates of C. elegans that were studied - that is, nearly all the lines are exclusively compatible with either the Bristol or the Hawaiian strain. Furthermore, the strains within each category share the same haplotype in the zeel-1/peel-1 interval.

The biological functions of zeel-1 and peel-1 are not clear, although zeel-1 shows homology to a subunit of an E3 ubiquitin ligase complex. The authors speculate that wild-type ZEEL-1 might counteract the toxic effect of paternally supplied PEEL-1.
The effect of a paternal-effect allele is intriguing in light of the fact that events early in embryogenesis are largely governed by maternal, not paternal, products.

From a population genetics perspective the challenge is to explain how the two incompatible zeel-1/ peel-1 haplotypes could be maintained over an estimated 3.5 million generations. This region is rather peculiar: the fact that it has accumulated more mutations than expected under neutrality is suggestive of balancing selection, which works to maintain genetic polymorphism.

The cause of the balancing selection remains to be identified. For now, the working model is that the developmental incompatibility arises as a side effect of balancing selection. Because of the worm's largely selfing mode of reproduction, each zeel-1/peel-1 haplotype would be free to accumulate mutations: the fitness consequences of these new, potentially incompatible but as yet unchallenged variants would be uncovered by selection only rarely, that is, when they are outcrossed.

Tanita Casci

ORIGINAL RESEARCH PAPER Seidel, H. S. et al. Widespread genetic incompatibility in C. elegans maintained by balancing selection. Science 10 January 2008 (doi: 10.1126/science.1151107) FURTHER READING Antoshechkin, I. \& Sternberg, P.W. The versatile worm: genetic and genomic resources for Caenorhabditis elegans research. Nature Rev. Genet. 8, 518-532 (2007) 